\title{
Journal of IMAB
}

- Annual Proceeding (Scientific Papers) 2016

vol. 22, issue 1 (January - March) 


\title{
Journal of IMAB - Annual Proceeding (Scientific Papers) Editorial Board
}

\author{
Editor-in-chief: \\ Prof. Dr. Krassimir Metodiev \\ Deputy Editor-in-chief: \\ Assoc. prof. Dr. George Kyuchukov \\ Editors:
}

Prof. Dr. Angelina Kisselova-Yaneva (Responsible for part Oral\&Dental Medicine) Assoc. prof. Dr. Liana Gercheva (Responsible for part Medicine)

\section{Associate Editors:}

Prof. Dr. Bogdan Petrunov,

Prof. Dr. Bogomila Manevska,

Prof. Dr. Bojidar Popov,

Dora Peychinska,

Assoc. prof. Dr. Ivan Galabov,

Assoc. prof. Dr. Ivan Zlatarov,

Dr. Lyubomir Tzvetanov,

Paula Lazarova,

Assoc. prof. Dr. Pencho Kossev;

\section{International Members:}

Prof. Dr. Kurt G. Naber - Germany;

Prof. Dr. Mario Di Gioacchino - Italy;

Prof. Dr. Pio Conty - Italy;

Prof. Dr. Dieter Falkenhagen - Austria;

Prof. Dr. Ian Gould - UK;

Prof. Dr. Bulent Berkarda - Turkey;

Prof. Dr. Roman Kozlov - Russia;

Prof. Dr. Dan Engelhard - Israel;

Prof. Dr. Raphael Saginur - Canada;

\section{Part Medicine:}

Prof. Dr. Anelia Klissarova

Prof. Dr. Ara Kaprelian

Prof. Dr. Dimitar K. Gospodinov

Prof. Dr. Ekaterina Titianova

Assoc. prof. Dr. Galya Gancheva

Prof. Dr. Iskren Kotzev

Assoc. prof. Dr. Ivelina Yordanova

Assoc. prof. Dr. Mariana Arnaudova

Assoc. prof. Dr. Maya Danovska

Assoc. prof. Dr. Snejina Vassileva

Prof. Dr. Snezhanka T. Tisheva

Assoc. prof. Dr. Valentin Stoyanov

Prof. Dr. Zaharyi Krastev;

Dr. Katarina Wassilew - Germany

Prof. Dr. Goce Spasovski - Macedonia

Prof. Dr. Sonja Genadieva-Stavric -

Macedonia
Part Oral\&Dental Medicine:

Assoc. prof. Dr. Ani Beltcheva, Dr. Assya Krasteva,

Assoc. prof. Donka Kirova,

Prof. Dr. Elka Popova,

Assoc. prof. Dr. Hristina Lalabonova,

Assoc. prof. Dr. Hristina Mihailova,

Assoc. prof. Dr. Ilyana Stoeva,

Dr. Maria Dencheva,

Assoc. prof. Dr. Mariana Dimova,

Assoc. prof. Dr. Metodi Abadjiev,

Prof. Dr. Milena Peneva,

Dr. Nikolai Yanev.

Assoc. prof. Dr. Vladimir Panov,

Prof. Dr. Alexandru Petre - Romania

Dr. Bogdan Calenic - Romania

Prof. Dr. Slave Naumovski - Macedonia,

Executive Director:

Gospodin Peytchinski

\section{Address for correspondence:}

\section{Editor-in-chief:}

Prof. Dr. Krassimir Metodiev

Headquarters of International Medical Association

Bulgaria (IMAB); 55, M. Drinov str., 9002 Varna,

Bulgaria,

Tel./Fax: +359/52/634 107, 379 777;

Mobile: $+359 / 888712407$

E-mail: kr.metod@yahoo.com, kr.metod@gmail.com;

\section{Deputy Editor-in-chief:}

Assoc. prof. Dr. George Kyuchukov

E-mail: kyuchger@abv.bg;

\author{
Publishing house and Editorial office of \\ the Journal of IMAB: \\ "Peytchinski, Gospodin Iliev" ET, \\ compl. Droujba bl. 116, ap. 41; \\ 5806 Pleven, Bulgaria \\ Phone: +359/64 871 100, \\ E-mail: publisher_imab@abv.bg, or \\ publisher.imab@gmail.com,
}

Publisher: Gospodin Peytchinski

Mobile: +359/888 213675

Journal of IMAB - Annual Proceeding (Scientific Papers)

2016, vol. 22, issue 1, (January - March)

ISSN: 1312-773X; DOI: http://dx.doi.org/10.5272/jimab.2016221 